\title{
Phase Contrast MRI with Flow Compensation View Sharing (FCVS)
}

\author{
Da Wang ${ }^{1,2}$, Jiaxin Shao ${ }^{1}$, Stanislas Rapacchi ${ }^{1}$, Matthew J. Middione ${ }^{1,2}$, Daniel B. \\ Ennis ${ }^{1,2,3}$, and Peng $\mathrm{Hu}^{1,2}$ \\ ${ }^{1}$ Department of Radiological Sciences, David Geffen School of Medicine, University of California, \\ Los Angeles, CA, USA \\ 2Biomedical Physics Interdepartmental Graduate Program, University of California, Los Angeles, \\ CA, USA \\ ${ }^{3}$ Department of Bioengineering, University of California, Los Angeles, CA, USA
}

\begin{abstract}
Purpose-To develop and evaluate a technique for accelerating phase contrast MRI (PC-MRI) acquisitions without significant compromise in flow quantification accuracy.
\end{abstract}

\begin{abstract}
Methods-PC-MRI is commonly acquired using interleaved flow-compensated (FC) and flowencoded (FE) echoes. We hypothesize that the FC data, which represents background phase, does not change significantly over time. Therefore, we proposed to under-sample the FC data and use a view-sharing approach (FCVS) to synthesize a composite FC frame for each corresponding FE frame. FCVS was evaluated in a flow phantom and healthy volunteers and compared with a standard FCFE PC-MRI.
\end{abstract}

Results-The FCVS sequence resulted in an error of $0.0 \%$ for forward flow and $2.0 \%$ for reverse flow volume when compared to FCFE PC-MRI in a flow phantom. Measurements in the common carotid arteries showed that the FCVS method had $-1.16 \mathrm{~cm} / \mathrm{s}$ bias for maximum peak velocity and $-0.019 \mathrm{~mL}$ bias in total flow, when compared to FCFE with the same temporal resolution, but double the total acquisition time. These results represent $₫ .3 \%$ bias error in velocity and volumetric flow quantification.

Conclusion-FCVS can accelerate PC-MRI acquisitions while maintaining flow and velocity measurement accuracy when there is limited temporal variation in the FC data.

\section{Keywords}

phase contrast MRI; velocity encoding; view sharing; flow rate; flow quantification; temporal resolution; temporal footprint

*Correspondence to: Peng Hu, PhD, Department of Radiological Sciences, 300 UCLA Medical Plaza Suite B119, Los Angeles, CA 90095, penghu@mednet.ucla.edu. 


\section{INTRODUCTION}

Phase-contrast MRI (PC-MRI) is a well-established technique for quantification of blood flow velocity and volume. The typical implementation of PC-MRI for cardiovascular applications requires a cardiac phase-resolved acquisition with adequate spatial and temporal resolution. These requirements often result in relatively long acquisition times, especially for 4D flow PC-MRI, which appears to be promising for a number of applications $(1-2)$.

Techniques such as non-Cartesian sampling (3-4), parallel imaging (5-7) and compressed sensing (8-10) have been developed to accelerate PC-MRI acquisitions by taking advantage of the faster speed of non-Cartesian trajectories, the coil geometry, or the inherent sparsity of PC-MRI data. In a typical PC-MRI exam, two sets of images are acquired in an interleaved fashion as shown in Fig. 1a. These acquisitions typically include the FC and the FE data, or alternately positive and negative FE data sets (i.e. bipolar flow encoding). The flow velocity is encoded in the phase difference between the two images. The FC image, although required for calculating the background signal phase, typically takes the same amount of time as the FE acquisition, which effectively doubles the temporal footprint (i.e. the time span of the data used to reconstruct a single temporal frame) (11) of each temporal frame and decreases the temporal resolution (i.e. the time between two successive temporal frames) by $50 \%$. To address this problem, the shared velocity encoding (12) technique uses an interleaved bipolar flow encoding strategy such that the temporal resolution is effectively doubled compared to conventional PC-MRI; however, the temporal footprint of each frame remains the same as the conventional PC-MRI. Temporal resolution and temporal footprint are two important indices that govern, for example, the measurement accuracy of peak velocity, which is important for a number of clinical applications including the evaluation of carotid artery stenosis (13-14). Low temporal resolution is associated with underestimation of the peak velocity (12) and a long temporal footprint in PC-MRI also results in underestimation of the peak velocity under-estimation due to temporal averaging and blurring of the FE data.

In certain PC-MRI applications, such as the assessment of volumetric blood flow in the carotid arteries and the brain, where physiological motion is small, each temporal frame of FC data is not expected to change significantly assuming the background phase does not change significantly over time. In this regard, the conventional FCFE PC-MRI acquisition strategy is redundant since it acquires the FC data for each individual temporal frame and the FC data does not contain any flow-encoded information. In the present work, we propose a technique to accelerate PC-MRI by using sliding window temporal view sharing of the FC data (FCVS), hence improving both the temporal resolution and temporal footprint. Flow phantom and in vivo studies were performed to validate the accuracy of peak velocity and volumetric flow measurements using the proposed FCVS technique.

\section{METHODS}

Our institutional review board approved this study. Informed consent was obtained from all participants in this study. All the phantom and in vivo study were performed on a $1.5 \mathrm{~T}$ 
scanner with 6-channel body (flow phantom study) or head-neck (in vivo studies) coils (Avanto, Siemens, Erlangen, Germany).

\section{MRI Pulse Sequence}

For blood vessels such as the common carotid artery (CCA), or peripheral vessels, we hypothesize that the phase of the FC image in a typical PC-MRI scan does not change significantly during the cardiac cycle because it only reflects the background phase, which is not expected to change significantly over time. Therefore, in this work, we propose a PCMRI technique that uses sliding window View Sharing of the FC data (FCVS) as shown in Fig. 1. In our approach, the FC data is sampled much less frequently than the FE data as only the FE data contains the flow-encoded phase information. In the example shown in Fig. $1 \mathrm{~b}$, an FC k-space line is acquired after every five FE lines and hence the FC data is undersampled by a rate $\mathrm{R}_{\mathrm{FC}}=6$. To compensate for the under-sampled $\mathrm{FC}$ data, a sliding window view-sharing pattern is employed to synthesize a composite $\mathrm{FC}$ frame for each corresponding $\mathrm{FE}$ frame. For the case of $\mathrm{R}_{\mathrm{FC}}=6, \mathrm{FC}$ data from the three cardiac phases before the current frame and two cardiac phases after are included to form the composite FC data for the current frame. Due to the need for FC data acquisition, the FE data is slightly under-sampled at $\left(\mathrm{R}_{\mathrm{FC}}-1\right) / \mathrm{R}_{\mathrm{FC}}=1.2$ and this is overcome by using standard TGRAPPA (15). To calculate the GRAPPA kernel for the FE data, the nearest number of frames equal to $\mathrm{R}_{\mathrm{FC}}$ were averaged and used as the auto calibration signal. Additionally, a four by three kernel was used for TGRAPPA reconstruction. The traditional phase difference was then calculated between each acquired FE cardiac phase and its corresponding composite FC frame. In our approach, a longer temporal footprint is used to reconstruct FC images due to view sharing. However, this enables a shorter temporal footprint for the FE phases, which carry the flow information.

\section{Image Analysis}

All phase difference images throughout the cardiac cycle were calculated by multiplying the FC image by the complex conjugate of the FE image on a pixel by pixel basis, adding the complex results from all of the receiver channels, and taking the phase angle of the sum (16). ROI contours of the CCA were drawn based on complex difference DICOM images (i.e. FC-FE) and propagated to all cardiac phase difference images. The mean flow velocities and peak velocities for each time point were calculated based on the mean and maximum phase difference within the ROI. The maximum mean velocity and the maximum peak velocity were calculated from the mean flow velocity and peak velocity waveforms, respectively, at the time point of the peak during the cardiac cycle. The mean flow rate was calculated by multiplying the mean flow velocity within the ROI by the area of the ROI. The volumetric flow was calculated by integrating the mean flow rate over the cardiac cycle. In the retrospective in vivo study, the error of the proposed FCVS technique in mean flow velocity and peak velocity quantification when compared with the FCFE reference was calculated as the root mean square error of all the data points over the cardiac cycle. A paired t-test was used to compare flow measurements with a two-sided $\mathrm{P}<0.05$ indicating statistical significance. 


\section{Computer Simulation}

Computer simulations were performed to study the impact of temporal resolution and temporal footprint on the accuracy of peak velocity measurements. As shown in Fig. 2a, a $170 \mathrm{~ms}$ half-sinusoidal velocity waveform followed by zero velocity was designed to approximate systolic CCA waveform at a heart rate of 75 beats per min (800 ms R-R interval). TR was assumed to be $8.5 \mathrm{~ms}$ for FC and FE acquisitions. FCVS PC-MRI signal with views-per-segment $=2 \& 4$ were simulated for maximum peak velocity comparison. For PC-MRI, the maximum peak velocity measurement is also dependent on the temporal offset between the time point of true peak and the time point when PC-MR samples are taken. Therefore, the FCVS signal was simulated for a wide range of temporal offsets to study this effect.

\section{FC Phase Consistency Study}

The proposed approach is based on the hypothesis that the signal phase of the FC images does not change significantly over time for relatively stationary tissues because it represents a relatively stable background phase. To test this hypothesis, six healthy volunteers $(\mathrm{N}=6)$ were imaged using a $1.5 \mathrm{~T}$ scanner and a standard FCFE PC-MRI sequence, which used through-plane velocity encoding (VENC $=110 \mathrm{~cm} / \mathrm{s}$ ), $30^{\circ}$ flip angle, $260 \mathrm{~Hz} / \mathrm{Pixel}$ readout bandwidth, and $\mathrm{TE}_{\min }=5.22-6.22 \mathrm{~ms}, \mathrm{TR}_{\min }=8.5-9.6 \mathrm{~ms}$. Other imaging parameters used were: $256 \times 256$ acquired matrix and $170 \times 170 \mathrm{~mm}^{2}$ to $200 \times 200 \mathrm{~mm}^{2}$ field-of-view (FOV), and $5 \mathrm{~mm}$ slice thickness. ROIs within the CCAs were subsequently chosen for each subject to measure the change in the FC phase over the cardiac cycle on a pixel-by-pixel basis. As the complex signal's phase is studied, only data from a single coil channel with the highest SNR in the carotids area was used.

\section{Retrospective In Vivo Study}

The same six fully-sampled data sets acquired for FC phase consistency study was subsequently used to perform flow velocity and volume measurements. The FC k-space data in these data sets were decimated by the rate $\mathrm{R}_{\mathrm{FC}}=6$ to mimic an under-sampled $\mathrm{FC}$ acquisition. The view-sharing technique was used to generate the composite, but fully sampled FC, cardiac phases as described in Fig. 1b. In our approach, the under-sampling rate $\mathrm{R}_{\mathrm{FC}}$ can potentially affect the reconstruction quality and the resultant quantitative phase (i.e. velocity) estimates. Recently, a PC-MRI technique (FE only, or FEO) has been described (17) where the FC data is only acquired for the last cardiac cycle and the same FC data is shared among all the other FE cardiac phases. FEO effectively under-samples the FC data acquisition by a factor equal to the number of cardiac phases. To demonstrate the effect of using different $\mathrm{R}_{\mathrm{FC}}$ and using the FEO approach, the calculated velocity and total volumetric flow based on $\mathrm{R}_{\mathrm{FC}}=6 \& 10$ using our FCVS technique and the FEO technique were compared with the ground truth from the fully-sampled PC-MRI data.

\section{Flow phantom study}

The FCVS sequence was implemented and tested prospectively using a flow phantom. The FCVS pulse sequence was compared to the standard FCFE PC-MRI sequence in order to demonstrate that it does not introduce significant errors into the volumetric flow and 
velocity measurements. A pulsatile, but reproducible flow waveform was generated with a computer controlled flow pump and used to test the agreement of the two sequences with the same temporal resolution. The phantom consisted of rigid pipe with a $25 \mathrm{~mm}$ inner diameter and $27 \mathrm{~mm}$ outer diameter connected by flexible tubes to a CardioFlow 1000MR computer controlled displacement pump (Shelley Medical Imaging Technologies, Toronto, Ontario, Canada). The rigid pipe was oriented along the length of the magnet bore and passed through an axial imaging slice at the magnet's isocenter. The flow pump generated a sinusoidal flow waveform with a peak flow rate of $290 \mathrm{~mL} / \mathrm{s}$ and a period of $924 \mathrm{~ms}$. A simulated retrospective ECG signal was used with $924 \mathrm{~ms}$ cardiac cycle length to synchronize data acquisition with the gated PC-MRI sequences. Data were acquired using standard FCFE PC-MRI sequence and the FCVS sequence. Both sequences used throughplane velocity encoding $(\mathrm{VENC}=150 \mathrm{~cm} / \mathrm{s}), 30^{\circ}$ flip angle, 1 (FCFE) or 2 (FCVS) view(s)per-segment, and $501 \mathrm{~Hz} / \mathrm{Pixel}$ readout bandwidth. Other parameters for the flow phantom study were: TE $=4.05 \mathrm{~ms}, \mathrm{TR}=8.25 \mathrm{~ms}, 384 \times 252$ acquired matrix, and a $319 \times 209 \mathrm{~mm}^{2} \mathrm{FOV}$, which resulted in in-plane resolution of $0.83 \times 0.83 \mathrm{~mm}^{2}$ with $5 \mathrm{~mm}$ slice thickness. The temporal resolution for both sequences were $16.5 \mathrm{~ms}$. Due to the acceleration by FCVS, the total acquisition time of the FCVS sequence was 50\% of the standard FCFE.

\section{Prospective In Vivo Study}

All scans were acquired during free breathing with retrospective ECG gating. The six volunteers recruited in the retrospective in vivo study were scanned again using the standard FCFE PC-MRI sequence and the prospective FCVS sequence. Both sequences were implemented with through-plane velocity encoding (VENC $=110 \mathrm{~cm} / \mathrm{s}$ ), $30^{\circ}$ flip angle, 260 $\mathrm{Hz} / \mathrm{Pixel}$ readout bandwidth, $\mathrm{TE}_{\min }=5.22-6.22 \mathrm{~ms}$, and $\mathrm{TR}_{\min }=8.5-9.7 \mathrm{~ms}$. Other imaging parameters included: $256 \times 256$ acquired matrix and $170 \times 170-200 \times 200 \mathrm{~mm}^{2} \mathrm{FOV}$, and 5 $\mathrm{mm}$ slice thickness. Four data sets were acquired in each volunteer: 1) FCVS with 4 viewsper-segment; 2) Standard FCFE with 2 views-per-segment; 3) FCVS with 2 lines views-persegment; 4) Standard FCFE with 1 view-per-segment. ROI contours for the left and right CCA were drawn for each volunteer and used to compare the mean flow velocity, peak velocity and total volumetric flow between FCVS and FCFE.

As both FCVS and recently proposed Shared Velocity Encoding (SVE) technique increase the temporal resolution of PC-MRI, six additional healthy volunteers were scanned to compare FCVS and SVE. A modified sequence based on the standard bi-polar flow encoding PC-MRI sequence was developed, where the positively and negatively flowencoded acquisitions are interleaved to enable the sliding-window SVE reconstruction. For each of the six volunteers, our prospective FCVS sequence and the modified bi-polar SVE PC-MRI sequence were performed in a randomized order. Specifically the following 5 data sets were acquired on each volunteer: 1) SVE with 4 views-per-segment; 2) FCVS with 4 views-per-segment; 3) SVE with 2 views-per-segment; 4) FCVS with 2 views-per-segment; 5) Standard FCFE with 1 view-per-segment. The measurements from the standard FCFE with 1 view-per-segment were used as reference. 


\section{RESULTS \\ Computer Simulation}

Fig. $2 \mathrm{~b}$ shows the simulated maximum peak velocity measurements as a function of the temporal offset between the PC-MRI sample points and the true peak. As expected, due to the finite sampling window, the maximum peak velocity is underestimated. Depending on the temporal shift between true peak and FCVS sampling point, the error for maximum peak velocity measurement varied between $0.3 \%$ and $1.5 \%$ for views-per-segment $=2$, and between $1.5 \%$ and $6.3 \%$ for views-per-segment $=4$. The relative percentage error was a function of TR, pulse duration, segment, temporal resolution and temporal footprint, but was independent of actual maximum peak velocity.

\section{FC Phase Consistency Study}

A pixel by pixel map (Fig. 3a) shows the standard deviation of the FC signal phase from the receiver channel with the highest SNR acquired on one healthy volunteer. The average standard deviation values within the ROIs for the left and right CCA both were $0.02 \pi$. Fig. $3 \mathrm{~b}$ shows the FC signal phase over time for one of the pixels within the ROI.

\section{Retrospective In Vivo Study}

The maximum mean flow velocity, peak velocity and total volumetric flow errors introduced by using view sharing with $\mathrm{R}_{\mathrm{FC}}=6$ and $\mathrm{R}_{\mathrm{FC}}=10$, and the $\mathrm{FEO}$ technique is shown in Table 1 . The view sharing technique with $\mathrm{R}_{\mathrm{FC}}=6$ resulted in significantly reduced errors in the measured maximum mean velocity, peak velocity and total volumetric flow compared to FEO ( $\mathrm{P}<0.05)$. The $\mathrm{R}_{\mathrm{FC}}=6$ acquisition had significantly reduced error in the measured maximum mean velocity compared to the $\mathrm{R}_{\mathrm{FC}}=10$ acquisition $(\mathrm{P}<0.05)$. The average percent error amongst all six subjects for the velocity calculations from $\mathrm{R}_{\mathrm{FC}}=6$ were $<2 \%$, and $<1 \%$ for total volumetric flow volume. Only one in 36 measurements from the $\mathrm{R}_{\mathrm{FC}}=10$ data had a percent error larger than 5\%. For FEO, 9 out of total 36 measurements were $>5 \%$. Based on these results, $\mathrm{R}_{\mathrm{FC}}=6$ was used for the prospective in vivo study.

An example of the measured mean and peak velocity waveforms over the cardiac cycle for one healthy volunteer is shown in Fig. 4. The velocity measurements based on view sharing $\left(\mathrm{R}_{\mathrm{FC}}=6\right)$ correlates well with the reference, with root mean square error of $0.77 \mathrm{~cm} / \mathrm{s}$ for mean flow velocity and $1.49 \mathrm{~cm} / \mathrm{s}$ for peak velocity when compared to the fully-sampled FCFE reference data. The FEO technique had a root mean square root error of $1.80 \mathrm{~cm} / \mathrm{s}$ for mean flow velocity and $3.69 \mathrm{~cm} / \mathrm{s}$ for peak velocity.

\section{Flow Phantom Study}

Mean flow velocities within a ROI measured by the prospective FCVS sequence were compared to measurements acquired with standard FCFE PC-MRI using the same temporal resolution, but twice the total acquisition time. The mean flow velocities over time were highly correlated (Fig. 5a). The total forward volumetric flow volume was $40.8 \mathrm{~mL}$ using FCVS and $40.8 \mathrm{~mL}$ using standard FCFE PC-MRI. The total reverse volumetric flows were $-29.6 \mathrm{~mL}$ for FCVS and $-29.0 \mathrm{~mL}$ for standard FCFE PC-MRI method. The forward and

Magn Reson Med. Author manuscript; available in PMC 2016 February 01. 
reverse percent error using the FCFE as the reference was $0.0 \%$ and $2.0 \%$, respectively. Fig. $5 \mathrm{~b}$ shows the peak velocity waveforms for the two sequences.

\section{Prospective In Vivo Study}

An example of mean flow velocity and peak velocity waveform measurements obtained on a healthy volunteer with: 1) FCFE PC-MRI with 2 views-per-segment and $34 \mathrm{~ms}$ temporal resolution, 2) FCVS with 2 views-per-segment and $17 \mathrm{~ms}$ temporal resolution, and 3) FCFE PC-MRI with 1 view-per-segment with $17 \mathrm{~ms}$ temporal resolution are shown in Fig. 6 . The $34 \mathrm{~ms}$ temporal resolution FCFE scan failed to capture the maximum peak velocity at around $75 \mathrm{~ms}$ into the cardiac cycle. The $17 \mathrm{~ms}$ FCVS scan provided similar peak velocity values compared to the $17 \mathrm{~ms}$ FCFE scan albeit at half of the total acquisition time. The maximum mean flow velocities and maximum peak velocity for the left and right CCA from the six volunteers are shown in Fig. 7., where the FCVS technique is compared with FCFE PC-MRI with the same temporal resolution ( $17 \mathrm{~ms})$, but twice the acquisition time. A Bland-Altman plot demonstrates that the bias was $0.03 \mathrm{~cm} / \mathrm{s}$ and the $95 \%$ confidence interval was $[-5.10,5.17] \mathrm{cm} / \mathrm{s}$ for the maximum mean flow velocity. The bias was -1.16 $\mathrm{cm} / \mathrm{s}$ and the $95 \%$ confidence interval was $[-6.13,3.81] \mathrm{cm} / \mathrm{s}$ for the maximum peak flow velocity. The total volumetric flow values measured in the left and right CCA in the six volunteers using FCVS with two different temporal resolutions of $17 \mathrm{~ms}$ and $34 \mathrm{~ms}$ (viewsper-segment $=2 \& 4$, respectively) and standard FCFE PC-MRI (temporal resolution=17 ms, views-per-segment=1) are shown in Fig. 8. For views-per-segment=4, the bias was 0.045 $\mathrm{mL}$ and the $95 \%$ confidence interval was $[-0.66,0.75] \mathrm{mL}$. For views-per-segment $=2$, the bias was $-0.019 \mathrm{~mL}$ and the $95 \%$ confidence interval was $[-0.20,0.24] \mathrm{mL}$.

Examples of peak velocity waveform comparisons obtained on a healthy volunteer with: a) FCVS (4 views-per-segment), SVE (4 views-per-segment) and FCFE (1 view-per-segment); and b) FCVS (2 views-per-segment), SVE (2 views-per-segment) and FCFE (1 view-persegment) are shown in Fig. 9. The maximum peak velocity of FCFE with 1 view-persegment was $95.0 \mathrm{~cm} / \mathrm{s}$, which was used as the reference. Maximum peak velocities measured by FCVS $(90.5 \mathrm{~cm} / \mathrm{s}$ and $4.7 \%$ error for 4 views-per-segment, and $96.0 \mathrm{~cm} / \mathrm{s}$ and $1.1 \%$ error for 2 views-per-segment) were more accurate than SVE $(80.2 \mathrm{~cm} / \mathrm{s}$ and $15.6 \%$ error with 4 views-per-segment, $87.4 \mathrm{~cm} / \mathrm{s}$ and $8.0 \%$ error with 2 views-per-segment). Based on data from the six subjects scanned, the average (range) percentage errors were $2.24 \%$ $(0.03 \%-5.62 \%)$ for FCVS (2 views-per-segment), 6.08\% (1.04\%-11.71\%) for SVE (2 views-per-segment), 3.74\% (0.11\%-9.03\%) for FCVS (4 views-per-segment), and 9.84\% (3.83\%-25.72\%) for SVE (4 views-per-segment).

\section{DISCUSSIONS}

In this work, we propose the FCVS technique for accelerating PC-MRI by under-sampling the FC k-space and performing a view-shared sliding window reconstruction of the FC data. This was feasible because the FC data only represent background phase that does not contain dynamic flow encoding information and hence are not expected to vary significantly over time, as demonstrated in Fig. 3. Our strategy results in a two-fold acceleration compared to standard FCFE PC-MRI while maintaining accuracy of in vivo blood flow 
velocity and volume measurements. Therefore, the proposed technique appears to be promising for flow quantification of blood vessels where there is no significantly physiological motion, such as carotids arteries, intracranial and peripheral blood vessels.

Similar techniques for performing view-sharing of the FC data have been previously applied to PC-MRI of the thorax (18) whereas we focus on the CCAs and potentially extend to intracranial or peripheral blood vessels. Many of the blood vessels in the thorax are subject to significant physiological motion and variations in vessel diameter due to the pulsatility of the blood flow. As a result, the FC phase for thoracic vessels likely has greater temporal variation compared to the carotid arteries. Therefore, further studies are needed to study the applicability of FCVS in the thorax and abdomen. Compared to the previous study in Ref. (18), our study provides a more detailed validation using both retrospective and prospective PC-MRI data, which can potentially bring the FC view-sharing techniques presented in our study and in Ref (18) closer to clinical utility.

The FCVS technique accelerates the PC-MRI acquisition by taking advantage of an inherent property of the background phase in PC-MRI. In this work, we demonstrate its utility in PCMRI with FE only in one direction. The same technique could potentially be applied to 4D PC-MRI where three FE directions are sampled. It is noted that the potential acceleration achievable for 4D PC-MRI is approximately 4/3-fold instead of 2-fold for 2D PC-MRI. Nevertheless, considering the often prohibitively long acquisition time of 4D PC-MRI, the 4/3-fold acceleration would likely achieve significant and useful savings in time. FCVS can be combined with other fast MRI techniques such as parallel imaging, compressed sensing or non-Cartesian readout to enable even faster PC-MRI sequence beyond what is achievable using other acceleration techniques alone. The extra acceleration could improve applications such as real-time single-directional flow encoding MRI.

The background phase of the FC data is not expected to change over time, but in practice, there might be slight variations for several reasons. Besides the aforementioned potential FC phase variation due to physiology motion in certain body regions, the flow related phase is not perfectly compensated for in the FC data due to small residual first (or second) order gradient moments, which might cause phase variations in the presence of pulsatile flow. Nevertheless, in our study, the phase variations in the FC data were all minimal, similar to Figure 3.

Recently reported shared velocity encoding (SVE) approach effectively doubles the temporal resolution of PC-MRI by using a bi-polar flow encoding strategy and a sliding window reconstruction technique. Although SVE, FEO and FCVS can all be used to increase the temporal resolution, there are important differences between them. Although SVE doubles the effective temporal resolution of conventional PC-MRI, its temporal footprint is twice as long as the temporal resolution because both the positive FE and negative FE data carry flow encoding information. Therefore, although the temporal frame rate is doubled in SVE, the estimated velocity in each temporal frame is the mean velocity averaged over two temporal frames, which results in underestimation of maximum peak velocity, as shown in Fig. 9. FEO only acquires the FC data for the last cardiac cycle and the same FC data is shared among all the other FE cardiac phases. FEO effectively improve the 
temporal resolution and temporal footprint by a factor of 2. However, as shown in Fig. 3b, even though FC data is relative consistent over time, it still has slight temporal variations. Any errors in the last FC frame will be propagated to all other FE temporal frames, which may result in errors on both peak velocity and volumetric flow estimations. Furthermore, although there is not significant bulk motion at the carotid arteries, it is possible that the carotid vessel diameter can expand or shrink slightly during the cardiac cycle. This can at least partially compensated for using our sliding window FC approach, but is expected to cause greater error using the FEO approach, which may explain why our FCVS approach has better flow estimation accuracy than FEO as shown in Fig. 4a. In FCVS, the temporal footprint of the $\mathrm{FC}$ frames is much longer than the FE frames due to the sliding window view sharing; however, only the FE echoes carry flow-encoding information. Therefore, the temporal footprint of FCVS is defined by the temporal footprint of the FE data, which is the same as the temporal resolution. Therefore, our FCVS approach improves both the temporal resolution and temporal footprint, which is of clinical importance for accurate velocity and flow quantification. However, FCVS has limitations. Since FCVS relies on ECG gating, its application in patients with arrhythmia can be limited, although the same limitation is also true for the conventional PC-MRI as well as the SVE and FEO approaches. Furthermore, when more views are sampled per k-space segment in FCVS, the temporal footprint of the FC data will inevitably become longer, which increases errors for both maximum peak velocity and volumetric flow measurements. In our study, the flow measurement bias errors associated with views-per-segment=4, which is commonly used in PC-MRI, were still relatively small.

\section{ACKNOWLEDGEMENT}

Research reported in this publication was supported by the National Heart, Lung, and Blood Institute of the National Institutes of Health under Award Number 1R21 HL113427 and the American Heart Association under the Award Number 10SDG4200076. The content is solely the responsibility of the authors and does not necessarily represent the official views of the sponsors of this work.

\section{Reference}

1. Markl M, Frydrychowicz A, Kozerke S, Hope M, Wieben O. 4D flow MRI. J. Magn. Reson. Imaging. 2012; 36:1015-1036. [PubMed: 23090914]

2. Harloff A, Albrecht F, Spreer J, et al. 3D blood flow characteristics in the carotid artery bifurcation assessed by flow-sensitive 4D MRI at 3T. Magn. Reson. Med. 2009; 61:65-74. [PubMed: 19097219]

3. Gu T, Korosec FR, Block WF, Fain SB, Turk Q, Lum D, Zhou Y, Grist TM, Haughton V, Mistretta CA. PC VIPR: A High-Speed 3D Phase-Contrast Method for Flow Quantification and HighResolution Angiography. Am. J. Neuroradiol. 2005; 26:743-749. [PubMed: 15814915]

4. Negahdar M, Kadbi M, Tavakoli V, Heidenreich J, Amini AA. Comparison of Cartesian, UTE radial, and spiral phase-contrast MRI in measurement of blood flow in extracranial carotid arteries: normal subjects. 2013; Vol. 8672:86720A-86720A-9.

5. Griswold MA, Jakob PM, Heidemann RM, Nittka M, Jellus V, Wang J, Kiefer B, Haase A. Generalized autocalibrating partially parallel acquisitions (GRAPPA). Magn. Reson. Med. 2002; 47:1202-1210. [PubMed: 12111967]

6. Pruessmann KP, Weiger M, Scheidegger MB, Boesiger P. SENSE: Sensitivity encoding for fast MRI. Magn. Reson. Med. 1999; 42:952-962. [PubMed: 10542355]

7. Kim D, Dyvorne HA, Otazo R, Feng L, Sodickson DK, Lee VS. Accelerated phase-contrast cine MRI using k-t SPARSE-SENSE. Magn. Reson. Med. 2012; 67:1054-1064. [PubMed: 22083998] 
8. Lustig M, Donoho D, Pauly JM. Sparse MRI: The application of compressed sensing for rapid MR imaging. Magn. Reson. Med. 2007; 58:1182-1195. [PubMed: 17969013]

9. Tao Y, Rilling G, Davies M, Marshall I. Carotid blood flow measurement accelerated by compressed sensing: Validation in healthy volunteers. Magn. Reson. Imaging [Internet].

10. Kwak Y, Nam S, Akçakaya M, Basha TA, Goddu B, Manning WJ, Tarokh V, Nezafat R. Accelerated aortic flow assessment with compressed sensing with and without use of the sparsity of the complex difference image. Magn. Reson. Med. 2013; 70:851-858. [PubMed: 23065722]

11. Haider CR, Hu HH, Campeau NG, Huston J, Riederer SJ. 3D high temporal and spatial resolution contrast-enhanced MR angiography of the whole brain. Magn. Reson. Med. 2008; 60:749-760. [PubMed: 18727101]

12. Lin H-Y, Bender JA, Ding Y, Chung Y-C, Hinton AM, Pennell ML, Whitehead KK, Raman SV, Simonetti OP. Shared velocity encoding: A method to improve the temporal resolution of phasecontrast velocity measurements. Magn. Reson. Med. 2012; 68:703-710. [PubMed: 22139889]

13. Srichai MB, Lim RP, Wong S, Lee VS. Cardiovascular Applications of Phase-Contrast MRI. Am. J. Roentgenol. 2009; 192:662-675. [PubMed: 19234262]

14. Vanninen RL, Manninen HI, Partanen PL, Vainio PA, Soimakallio S. Carotid artery stenosis: clinical efficacy of MR phase-contrast flow quantification as an adjunct to MR angiography. Radiology. 1995; 194:459-467. [PubMed: 7824727]

15. Breuer FA, Kellman P, Griswold MA, Jakob PM. Dynamic autocalibrated parallel imaging using temporal GRAPPA (TGRAPPA). Magn. Reson. Med. 2005; 53:981-985. [PubMed: 15799044]

16. Lu K, Liu TT, Bydder M. Optimal phase difference reconstruction: comparison of two methods. Magn. Reson. Imaging. 2008; 26:142-145. [PubMed: 17572035]

17. Offerman, E.; Glielmi, C.; Markl, M.; Koktzoglou, I.; Edelman, RR. Proceedings of the 21st Annual Meeting of ISMRM. Salt Lake City, Utah, USA: 2013. Flow-Encode Only Phase Contrast Magnetic Resonance Imaging. Abstract 4434.

18. Lin, H-Y.; Ding, Y.; Chung, Y.; Raman, SV.; Simonetti, OP. Proceedings of the 15th Annual Meeting of ISMRM. Berlin, Germany: 2007. Efficient Velocity Mapping by Accelerated Acquisition of Phase Reference Data; p. 249 


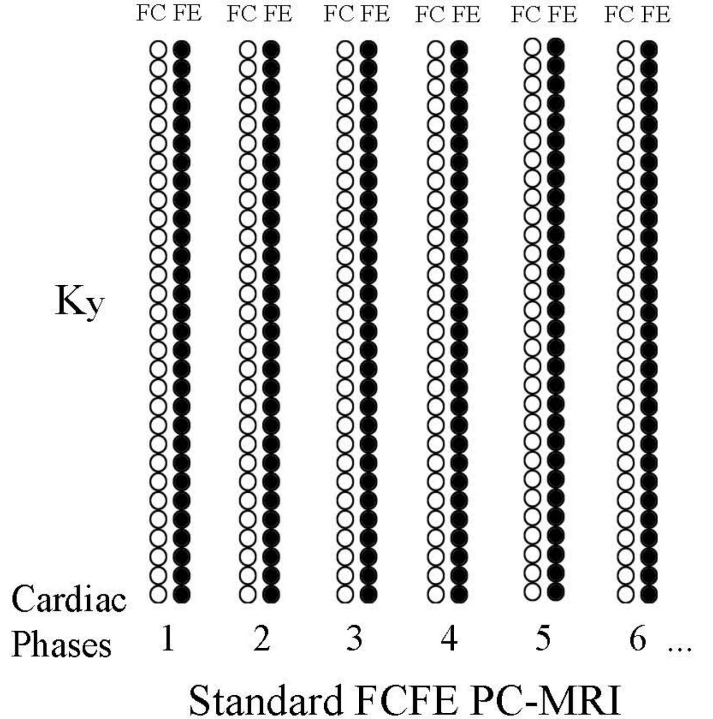

(a)

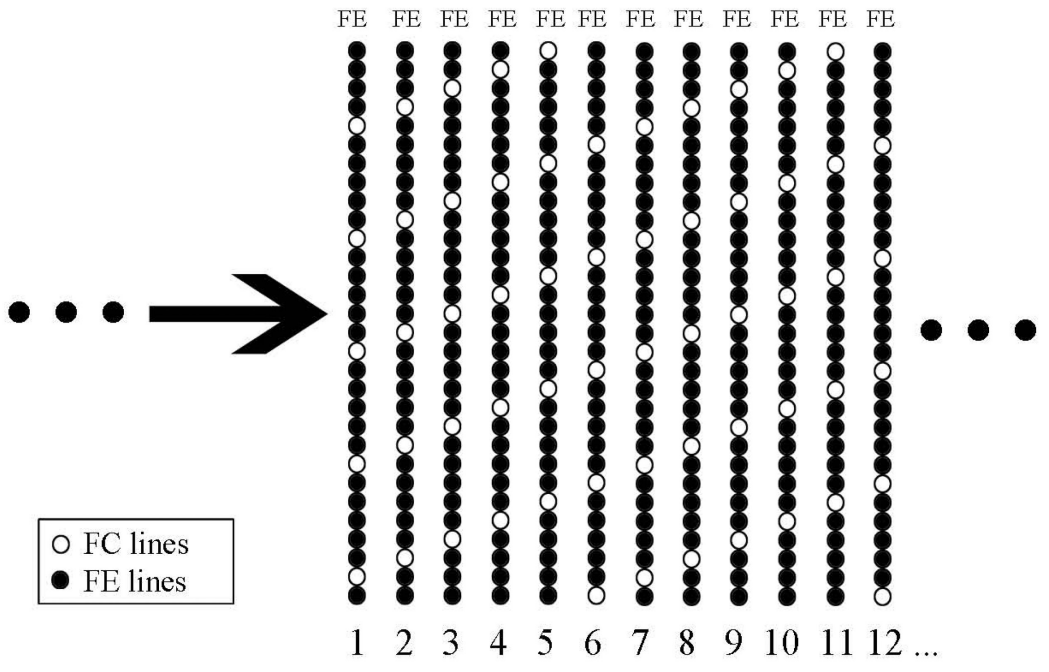

FCVS

(b)

Fig. 1.

The data acquisition scheme of (a) the standard FCFE PC-MRI and (b) the proposed FCVS approach. The FCVS approach approximately doubles the effective temporal resolution by under-sampling the FC data by a factor of six. 


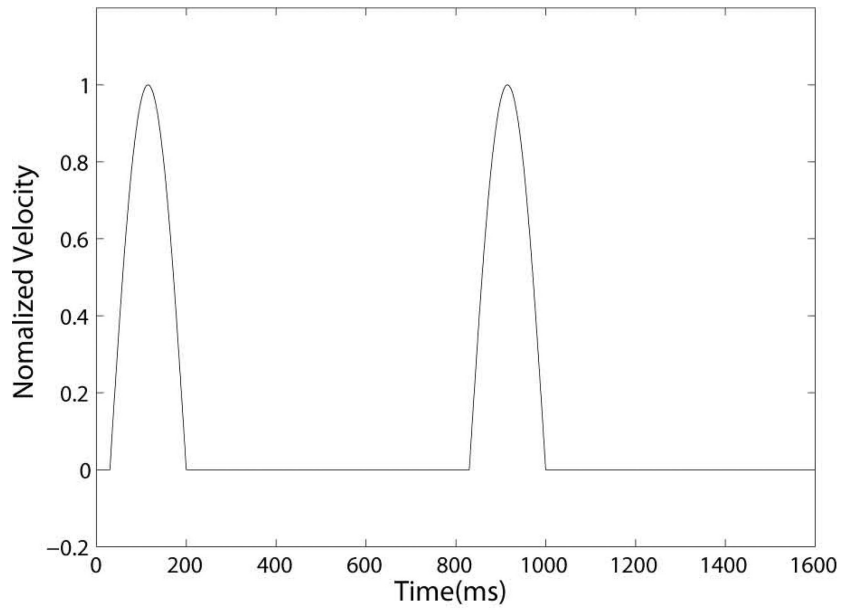

(a)

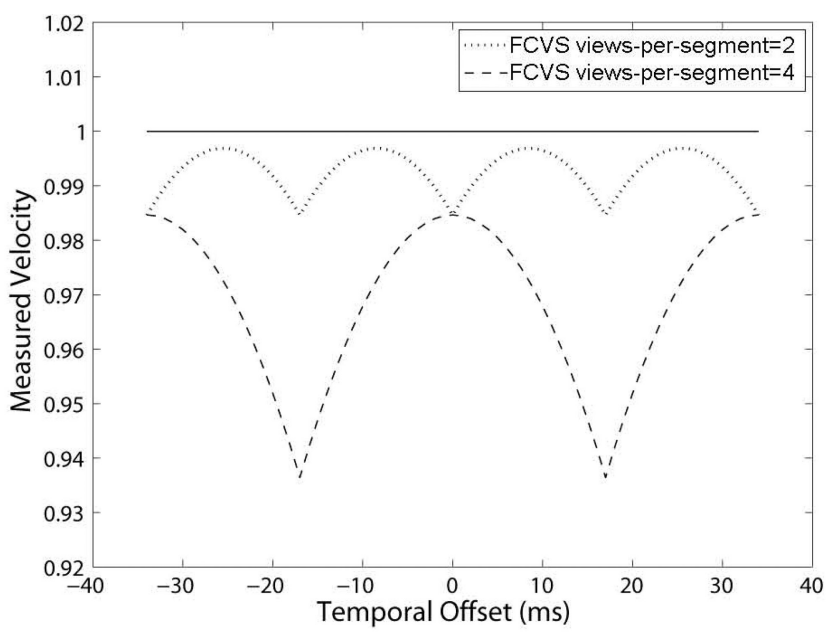

(b)

Fig. 2.

(a) Simulated CCA peak velocity waveform with $170 \mathrm{~ms}$ half-sinusoidal waveform and 800 ms R-R interval. (b) Theoretical simulation results show influence of temporal offset, temporal resolution and temporal footprint of FCVS with views-per-segment $=2 \& 4$ on the accuracy of maximum peak velocity measurements. 
rad
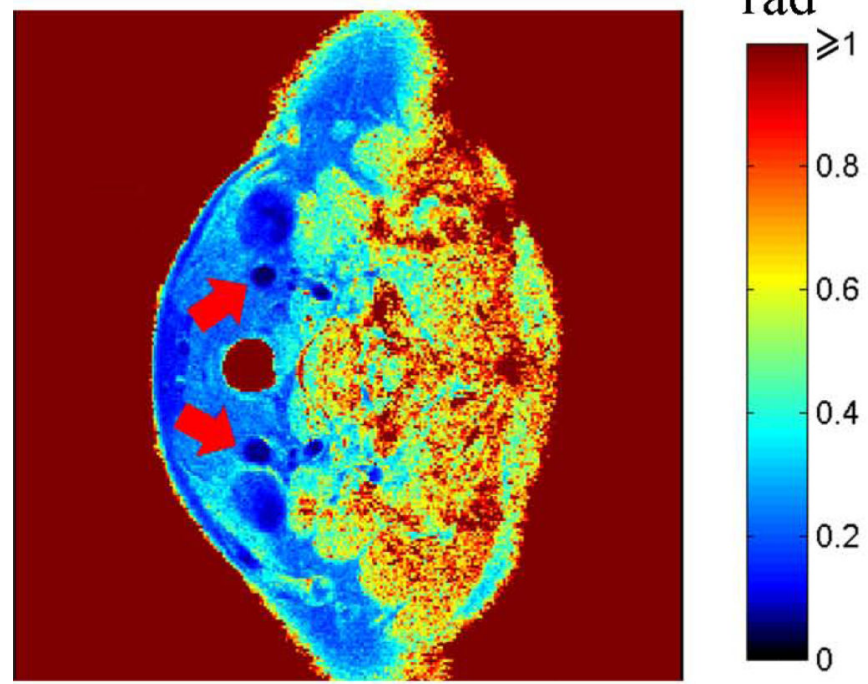

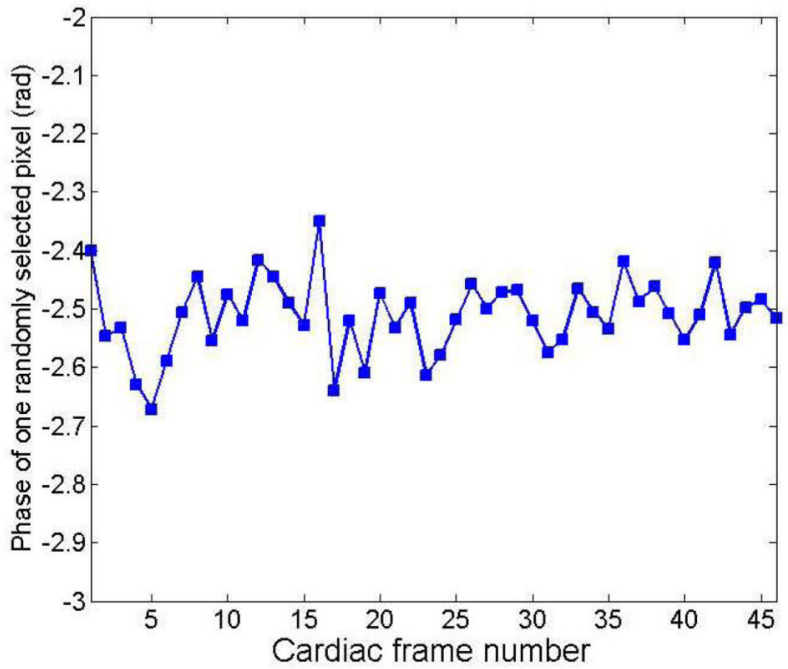

(b)

Fig. 3.

(a) A pixel by pixel map of the standard deviation of the FC data phase from a single receiver coil with highest SNR. CCAs (red arrows) had very small phase variation through time $(\mathrm{SD}= \pm 0.02 \pi)$. The large phase variation at locations far away from the coil was due to low SNR. (b) The FC signal phase as a function of the cardiac frames for a randomly selected pixel within the CCA (mean/ \pm SD: $-2.51 / \pm 0.065)$. 


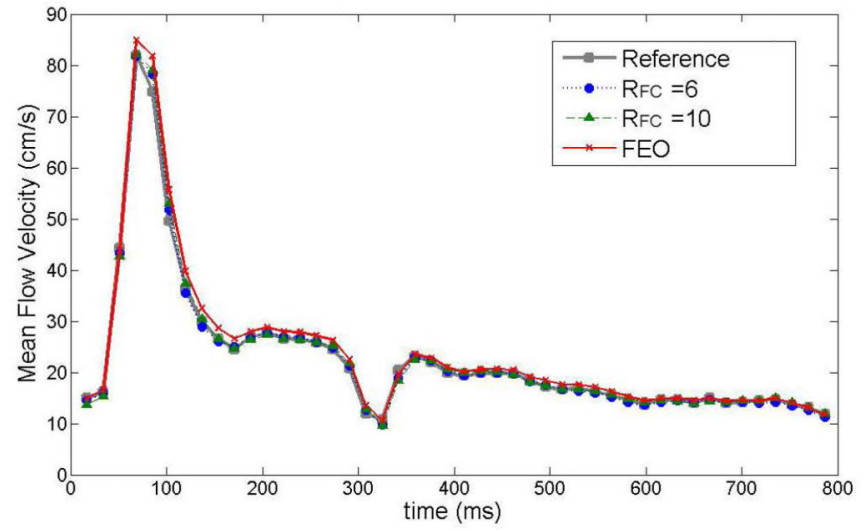

(a)

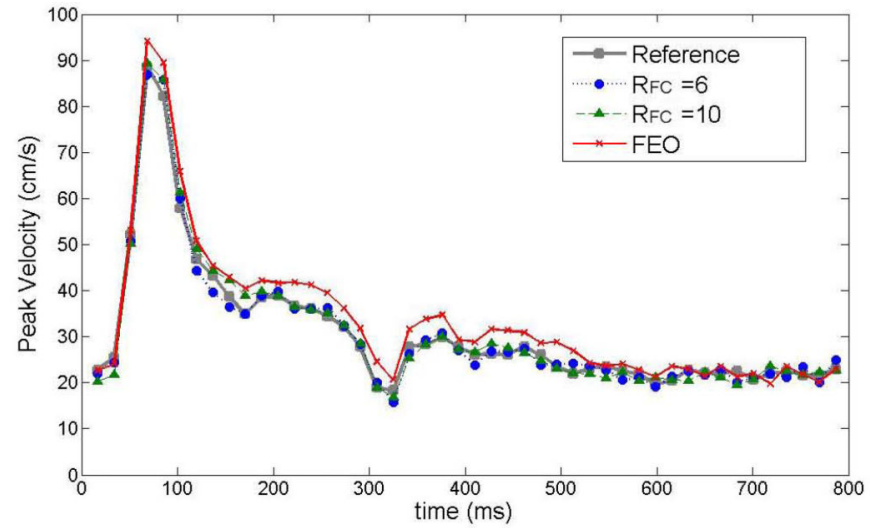

(b)

Fig. 4.

Comparison of (a) the mean flow velocity waveform and (b) the peak velocity waveform for left CCA measured by standard FCFE PC-MRI (gray curve), view sharing $\left(\mathrm{R}_{\mathrm{FC}}=6\right.$, blue curve, $\mathrm{R}_{\mathrm{FC}}=10$, green curve), and FEO PC-MRI (red curve). The view sharing technique provides accurate measurements of mean flow velocity and peak velocity. As the FC undersampling factor $\mathrm{R}_{\mathrm{FC}}$ increases from 6 to 10 and then approaches $\mathrm{FEO}$, the measurement accuracy decreases. In this example FEO overestimates the mean flow velocity and peak velocity. 


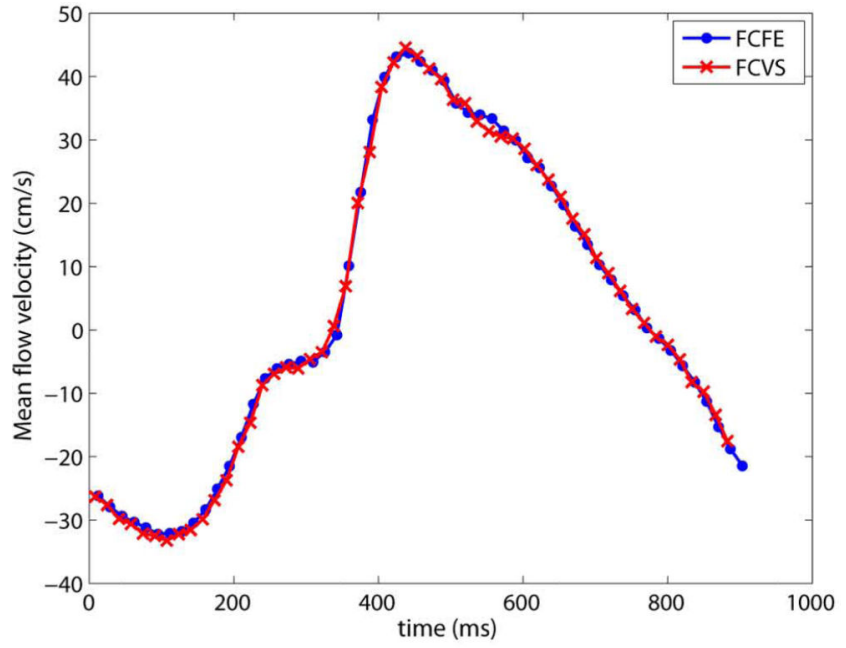

(a)

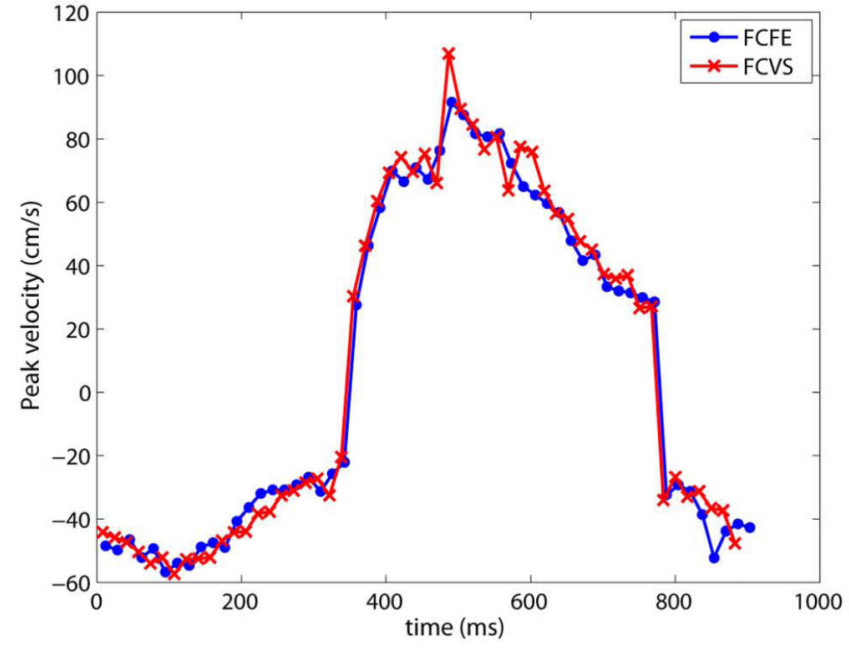

(b)

Fig. 5.

(a) Mean flow velocity waveform and (b) peak velocity waveform for the flow phantom study. FCVS (red curve) and standard FCFE PC-MRI (blue curve). The FCVS technique provides accurate flow velocity measurements albeit only using half of the acquisition time as standard FCFE PC-MRI. 


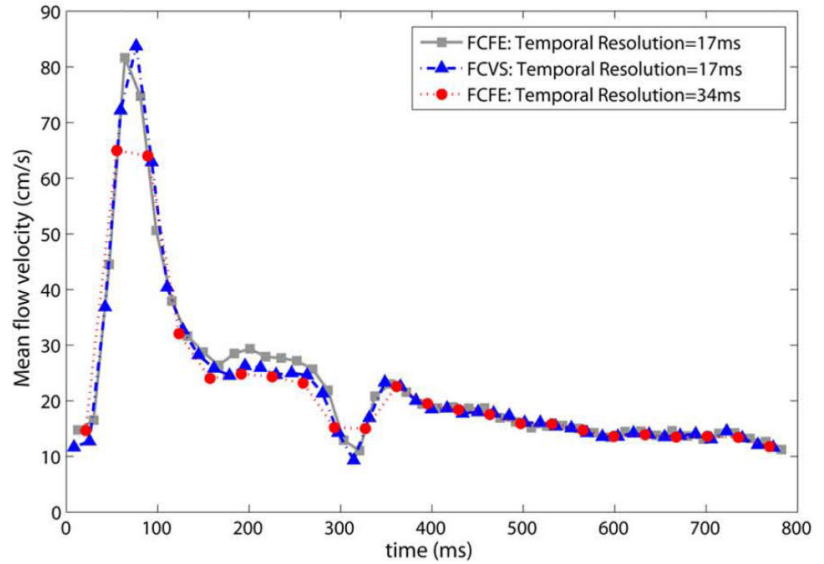

(a)

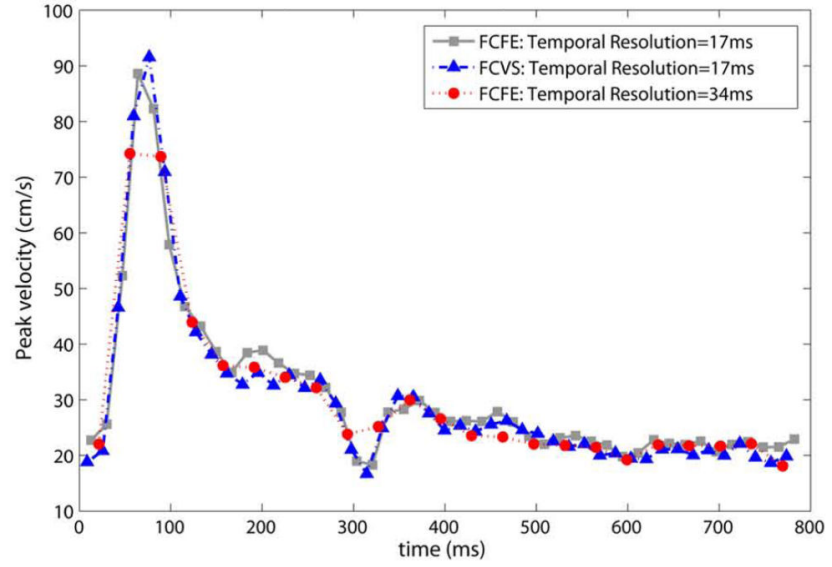

(b)

Fig. 6 .

(a) Mean flow velocity waveforms and (b) Peak velocity waveforms from the standard FCFE PC-MRI (gray curve) with temporal resolution=17 ms and acquisition time of $214 \mathrm{~s}$, FCVS (blue curve) with temporal resolution=17 $\mathrm{ms}$ and acquisition time of $106 \mathrm{~s}$, standard FCFE PC-MRI (red curve) with temporal resolution=34 $\mathrm{ms}$ and acquisition time of $107 \mathrm{~s}$. The FCVS results are highly correlated with the measurements from standard FCFE PCMRI at the same temporal resolution but FCVS only requires $50 \%$ of the acquisition time. The standard FCFE PC-MRI fails to capture the peak velocity at approximately $75 \mathrm{~ms}$ or the transient dip at $320 \mathrm{~ms}$ when its temporal resolution is halved to match the total acquisition time of FCVS. 


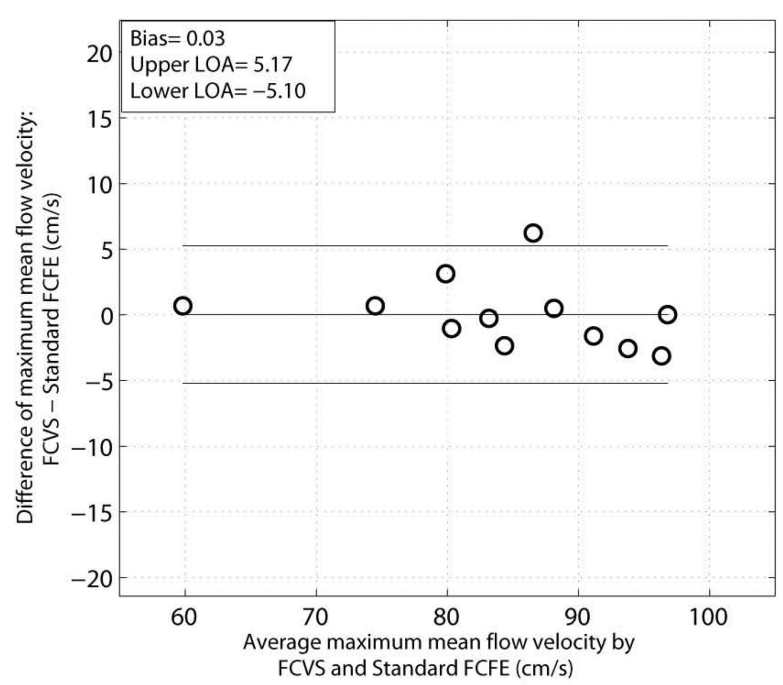

(a)

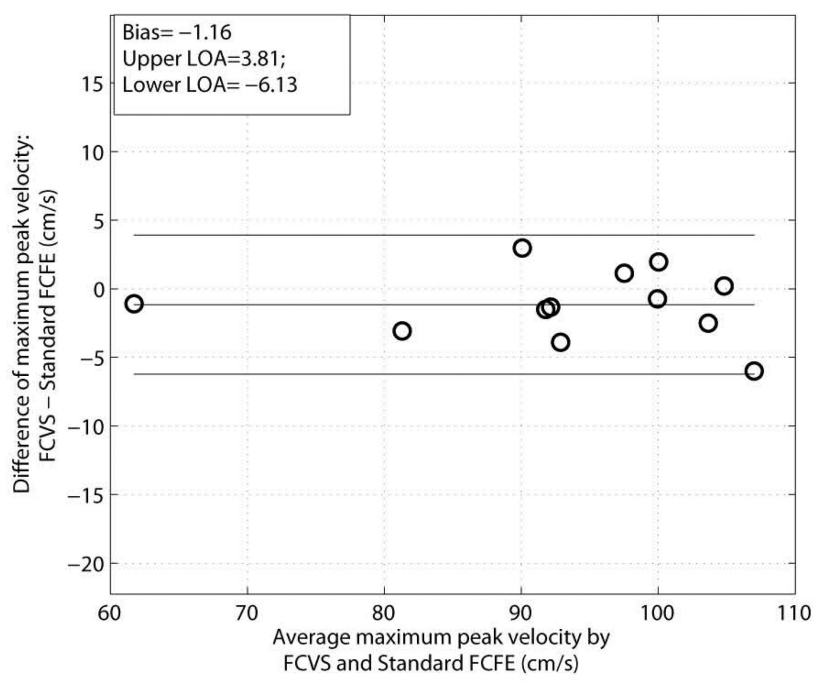

(b)

Fig. 7.

(a) The Bland-Altman plot of the maximum mean flow velocities between standard FCFE PC-MRI and FCVS, (b) The Bland-Altman plot of maximum peak velocities between standard FCFE PC-MRI and FCVS. The standard FCFE PC-MRI had the same temporal resolution as FCVS, but double the acquisition time. LOA :limits of agreement, interval between upper and lower LOA is also known as 95\%-CIs 


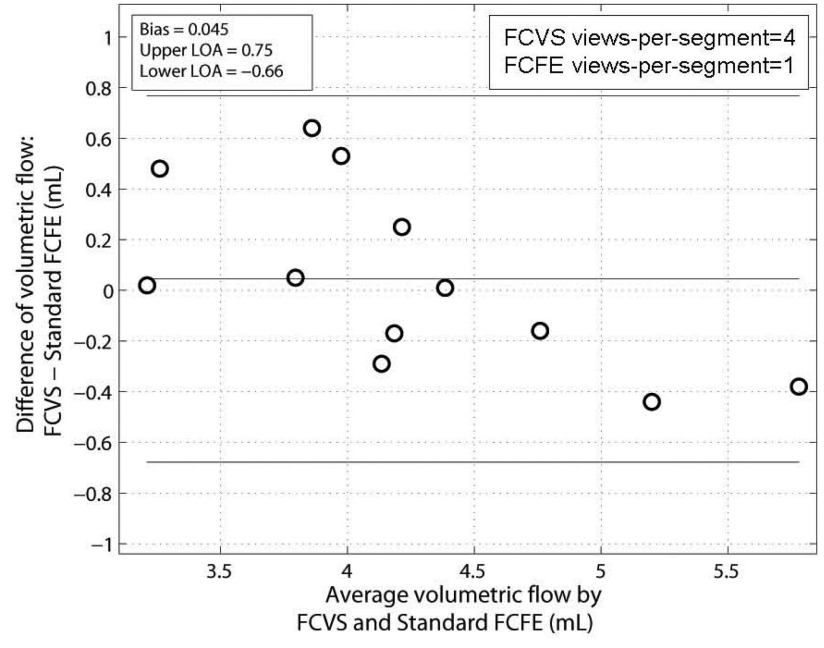

(a)

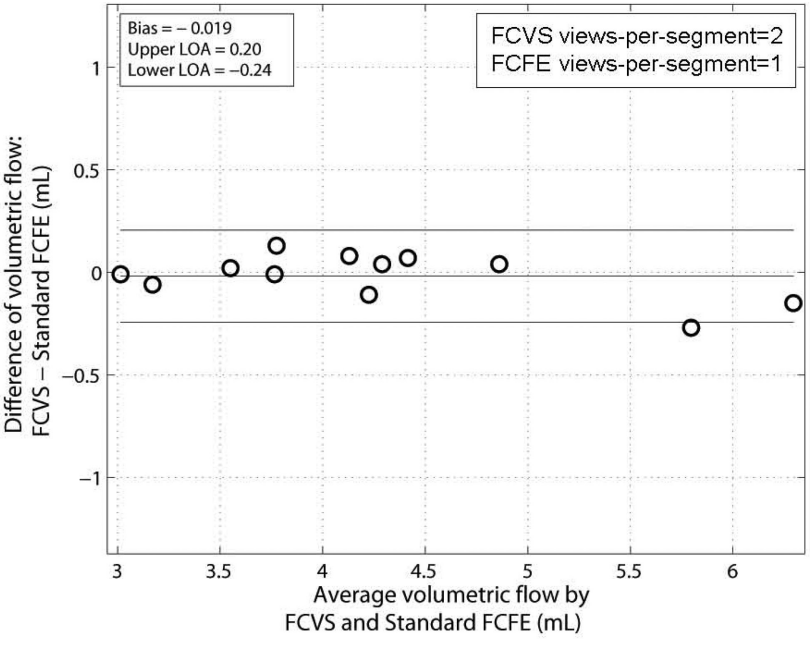

(b)

Fig. 8.

The Bland-Altman plot of total volumetric flow measurements between standard FCFE PCMRI (1 view-per-segment) and FCVS with two different temporal resolutions: in the left and right CCA in six volunteers for a total 12 flow measurements: a) 4 views-per-segment FCVS and 1 view-per-segment FCFE, b) 2 views-per-segment FCVS and 1 view-per-segment FCFE. In b), the standard FCFE PC-MRI had the same temporal resolution as FCVS, but double the acquisition time. 


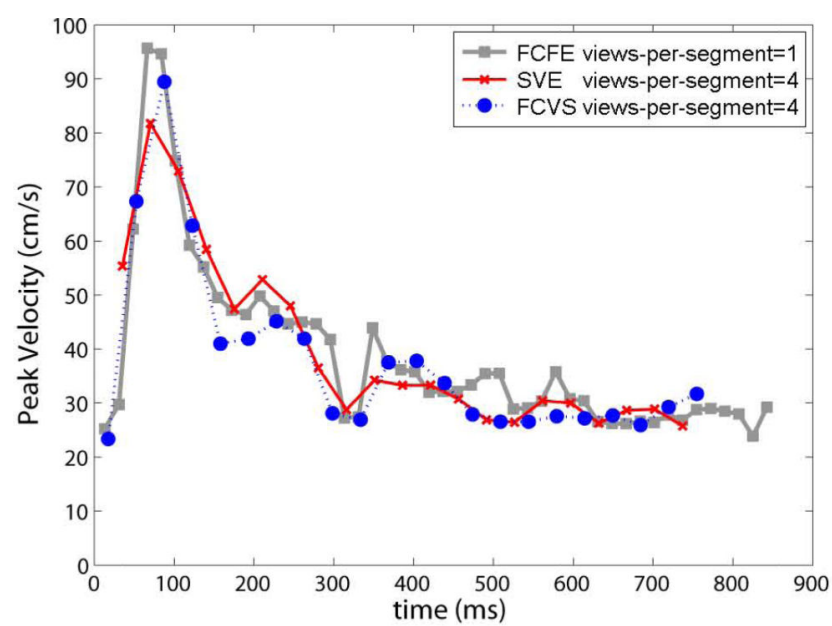

(a)

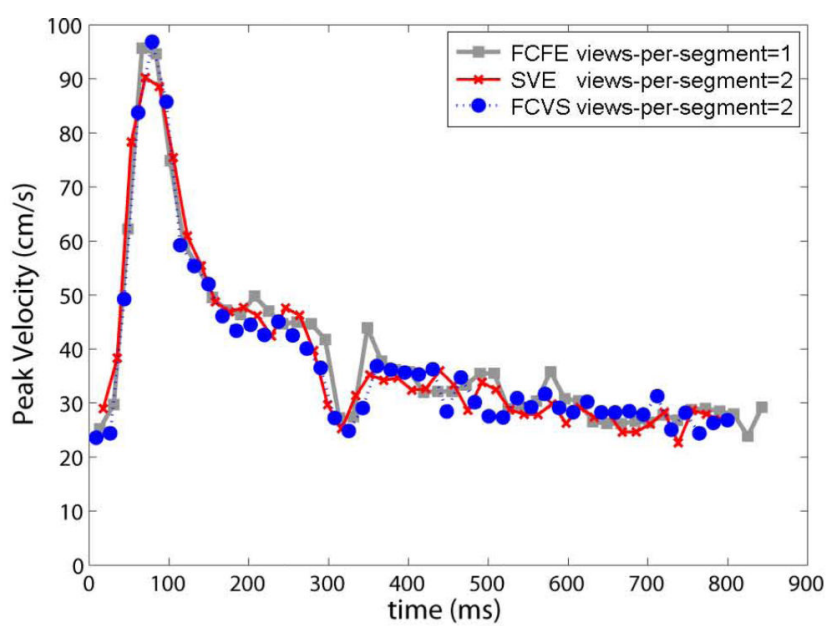

(b)

Fig. 9.

Example peak velocity waveforms from left CCA of one volunteer: (a) standard FCFE PCMRI (gray curve) with 1 view-per-segment, FCVS (blue curve) with 4 views-per-segment and SVE (red curve) with 4 views-per-segment, (b) standard FCFE PC-MRI (gray curve) with 1 view-per-segment, FCVS (blue curve) with 2 views-per-segment and SVE (red curve) with 2 views-per-segment. The SVE tends to underestimate the maximum peak velocity due to its longer temporal footprint. 


\section{Table 1}

Comparison of percent errors introduced by $\mathrm{FC}$ images view sharing with different $\mathrm{R}_{\mathrm{FC}}$, and using last $\mathrm{FC}$ frame for all phase difference calculation (FEO) in six volunteers.

\begin{tabular}{llll}
\hline & $\begin{array}{l}\text { Maximum mean flow } \\
\text { velocity error }\end{array}$ & $\begin{array}{l}\text { Maximum peak velocity } \\
\text { error }\end{array}$ & Total flow error \\
\hline $\mathrm{R}_{\mathrm{FC}}=6, \mathrm{LCCA}$ & $1.2 \%(0.2 \%-2.2 \%)$ & $1.7 \%(0.7 \%-2.3 \%)$ & $0.5 \%(0.2 \%-1.0 \%)$ \\
$\mathrm{R}_{\mathrm{FC}}=6, \mathrm{RCCA}$ & $1.0 \%(0.1 \%-1.5 \%)$ & $1.0 \%(0.1 \%-2.0 \%)$ & $0.3 \%(0.1 \%-0.4 \%)$ \\
\hline $\mathrm{R}_{\mathrm{FC}}=10, \mathrm{LCCA}$ & $2.1 \%(0.2 \%-3.4 \%)$ & $2.4 \%(0.3 \%-6.3 \%)$ & $0.7 \%(0.3 \%-1.5 \%)$ \\
$\mathrm{R}_{\mathrm{FC}}=10, \mathrm{RCCA}$ & $1.3 \%(0.6 \%-1.5 \%)$ & $1.4 \%(0.2 \%-2.3 \%)$ & $0.4 \%(0.2 \%-0.6 \%)$ \\
\hline $\mathrm{FEO}, \mathrm{LCCA}$ & $3.3 \%(0.1 \%-5.8 \%)$ & $3.9 \%(0.1 \%-6.5 \%)$ & $4.3 \%(2.3 \%-7.9 \%)^{*}$ \\
$\mathrm{FEO}, \mathrm{RCCA}$ & $2.0 \%(0.1 \%-6.4 \%)$ & $2.5 \%(0.9-\% 4.3 \%)$ & $3.5 \%(1.6 \%-5.7 \%)^{*}$ \\
\hline
\end{tabular}

P $<0.05$ when compared with $\mathrm{RFC}=6$ at the same location (LCCA or RCCA). LCCA: left common carotid artery; RCCA: right common carotid artery 